\title{
Alternative UV Light Sources for Surface Disinfection
}

\author{
Atis Skudra \\ Institute of Atomic Physics and \\ Spectroscopy \\ University of Latvia \\ Riga, Latvia \\ askudra@latnet.lv \\ Gita Rēvalde \\ Institute of Technical Physics \\ Riga Technical University \\ Riga, Latvia \\ gita.revalde@rtu.lv \\ Tālis Juhna \\ Water Research and Environmental \\ Biotechnology Laboratory \\ Riga Technical University \\ Riga, Latvia \\ talis.juhna@rtu.lv
}

\author{
Linda Mežule \\ Water Research and Environmental \\ Biotechnology Laboratory \\ Riga Technical University \\ Riga, Latvia \\ linda.mezule@rtu.lv \\ Anna Zajakina \\ Latvian Biomedical Research and \\ Study Centre \\ Riga, Latvia \\ anna@biomed.lu.lv
}

\author{
Karīna Spunde \\ Latvian Biomedical Research and \\ Study Centre, \\ Riga, Latvia \\ spunde.carina@gmail.com \\ Madara Ziṇge \\ Institute of Atomic Physics and \\ Spectroscopy \\ University of Latvia \\ Riga, Latvia \\ madara.zinge@lu.lv
}

\begin{abstract}
Mercury UV-C light sources are long known to be efficient for microbial inactivation and have been widely used. At the same time, the radiation, if used in inappropriate doses and spectral regimes, can also cause harmful effects to human tissue. The aim of the study was to evaluate the applicability of the novel UV light sources from thallium antimony at different UV-C. For the research specially made light sources were produced. The influence of UV-C radiation in the range of $200-280 \mathrm{~nm}$ was tested on Gramnegative bacterium Escherichia coli, both with mercury and thallium. More than $\mathbf{9 9 . 9 9} \%$ inactivation of $E$. coli cells was obtained after $10 \mathrm{~min}$ contact time for thallium - antimony UV-C light source, demonstrating the potential of the produced lamps.
\end{abstract}

Keywords - disinfection, UV thallium lamp, UV mercury lamp, UV-C, antiviral, antibacterial

\section{INTRODUCTION}

Ultraviolet radiation in the range of $200-280 \mathrm{~nm}$ (UVC) has long been known to have the abilities to cause damage to the cellular material of bacteria or viruses, including their DNA or RNA. The inactivation occurs when the absorption of a photon forms pyrimidine dimers between adjacent thymine bases and renders the microorganism incapable of replicating [1]. As reported [2], at appropriate doses UV-C can selectively inactivate microorganisms while preserving viability of mammalian cells and, moreover, promote wound healing.

The wide application of UV-C for disinfection purposes has been linked with multiple advantages over liquid disinfectants and heat sterilization. It can be performed to disinfect surfaces, liquids, air and rooms, and it is also very energy-efficient [3]. All these factors are of high importance not only in routine disinfection but also during local and global pandemics, e.g., SARS-CoV-2, swine flu, MERS-CoV.

The spectral region used for disinfection is mainly the UV-C radiation in the spectral range of $200-280 \mathrm{~nm}$. To produce radiation in the UV-C region, in almost all disinfection experiments mercury low-pressure lamps have been used with a strong emission line at $254 \mathrm{~nm}$ what is close to the RNA absorption peak [3]. There are very few experiments applying other wavelengths. Only individual 
experiments with a peak wavelength at $222 \mathrm{~nm}$, and 365 $\mathrm{nm}$ (UV-A) [3] or $207 \mathrm{~nm}$ [4] have been reported. One of the reasons for the predominant use of $\mathrm{Hg} 254 \mathrm{~nm}$ light is the widespread ability of $\mathrm{Hg}$ light sources due to simple manufacturing process and lack of other appropriate UV$\mathrm{C}$ light sources. This has so far been a limitation for exploitation of other UV-C wavelengths.

Thus, the aim of this study was to evaluate the applicability of the novel UV light sources filled with thallium and antimony for their potential to be used in disinfection. Thallium and antimony filling would give more working spectral lines in the same region. These wavelengths of the UV-C spectral region will be compared with mercury resonance line to demonstrate the efficiency.

Institute of Atomic Physics and Spectroscopy, University of Latvia has experience in development and manufacturing of special design high-frequency low pressure metal vapour light sources. Such light sources are widely used as bright radiators of intense spectral lines in different types of scientific devices, for instance, in atomic absorption spectrometers [5,6].

The high -frequency light sources are excited by the electromagnetic field, using electrodes outside the lamp. As the result, the inductive coupled discharge is initiated. It has been proven that such kind of discharge plasma is characterised by both, higher electron temperature and concentration, giving more intense spectral lines in comparison to other type of low pressure discharges.

Multiple high-frequency light sources, radiating in UV region filled with such elements like lead, phosphor, selenium, arsenic, thallium, antimony and mercury have been manufactured [7]. At the moment, these light sources have to be optimized for each particular use, and it is of great importance to use reliable methods for these investigations and further on for improvement of the light sources.

Within this work, we report the results of disinfection experiments with the thallium - antimony light sources in comparison with mercury lamps for neutralisation of Escherichia coli as representative Gramnegative bacterium.

\section{MATERIALS AND METHODS}

\section{A. UV-ligh sources}

For the experiment, special type high-frequency electrodeless light sources were manufactured at the Institute of Atomic Physics and Spectroscopy, University of Latvia. The light sources were made of $\mathrm{SiO}_{2}$ glass with a diameter of $10 \mathrm{~mm}$, filled with a metal vapour and a buffer gas at low pressure (Fig. 1).

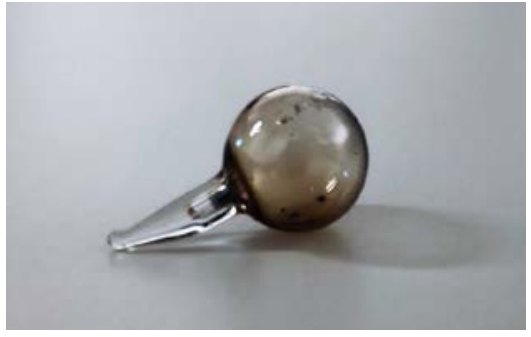

Fig. 1. Typical design of the high-frequency light source.

In this case, thallium and antimony mixture was used for filling to excite more spectral lines in the UV-C spectral region. As a buffer gas, argon was used at the pressure of 3 torr. An outer electromagnetic field of about $100 \mathrm{MHz}$ frequency was applied to initiate a discharge inside the lamp.

Spectra of the manufactured metal vapour light sources were recorded by the high - resolution Jobin Yvon 1000 M spectrometer.

\section{B. Bacterial culture and growth conditions}

Escherichia coli ATCC®10536 was used as the test bacterium. Overnight culture in Tryptone soya agar (Oxoid Ltd, UK) were thrice washed with sterile peptone water $(0.1 \%)$ by centrifugation (6000 rpm for 2 minutes, Minispin, Eppendorf). The final bacterial pellet was resuspended in sterile peptone water $(0.1 \%)$ to obtain a stock solution of approximately $10^{7}$ colony forming units (CFU) $\mathrm{mL}^{-1}$. For cell enumeration $0.002 \mathrm{~mL}$ of the stock suspensions were filtered through a 25-mm-diameter $0.2-$ $\mu \mathrm{m}$-pore-size filter (Polycarbonate Track- Etch Membrane, Sartorius, Germany) and fixed with 3-4\% formaldehyde for 10 minutes, washed with sterile distilled water and stained with $10 \mu \mathrm{g} \mathrm{mL} \mathrm{mL}^{-1}$ DAPI (4',6-diamidino2-phenylindole, Merck, Germany) for 5-10 minutes. Cell concentrations were determined with epifluorescence microscopy (Ex: 340/380; Em: > 425, dichromatic mirror 565 nm, Leica DM6000B, Germany) by counting of 20 random fields of view.

\section{Disinfection experiments}

To test the inactivation efficiency, $3 \mathrm{~mL}$ of $E$. coli stock was inserted in sterile $30 \mathrm{~mm}$ borosilicate Petri dish and placed under the lamp at the distance of $11 \mathrm{~cm}$. The $\mathrm{SiO}_{2}$ lens was used to create parallel light rays. The measurements were performed in several series, changing the irradiation time form $1 \mathrm{~min}$ to $10 \mathrm{~min}$. 


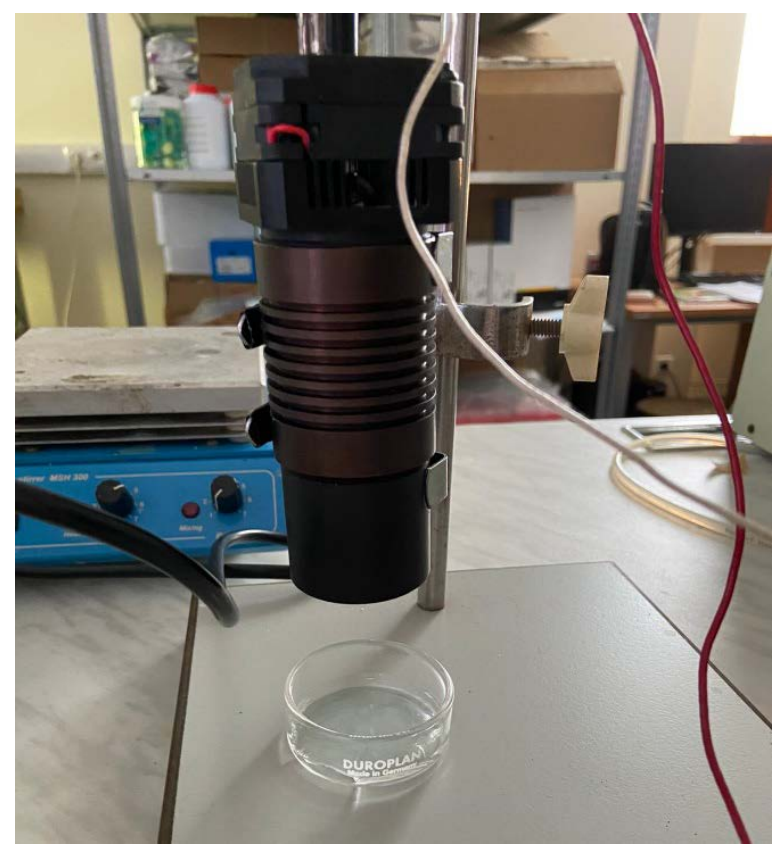

Fig. 2. Experimental set-up for irradiation tests.

Immediately after irradiation, the sample was removed from the light source and decimal dilutions of the sample were inoculated onto Tryptone soya agar (Oxoid Ltd, UK) plates and incubated for 24 hours at $37^{\circ} \mathrm{C}$. The result (reduction in cultivable E. coli) is expressed as negative log reduction of colony forming units after treatment divided by colony forming units before treatment.

\section{RESULTS AND DISCUSSION}

A design of a high - frequency thallium - antimony light source is shown in Fig.3.

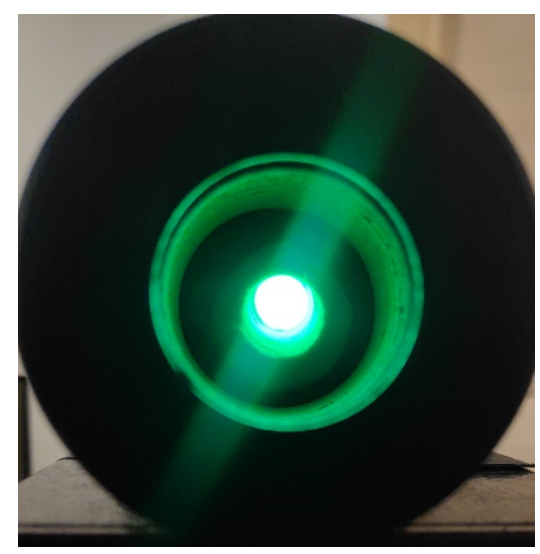

Fig. 3. Design of a high-frequency thallium - antimony metal vapour light source

UV- C spectra of thallium-antimony and mercury light sources are shown in Fig.4 and Fig. 5, respectively. As can be seen, the mercury UV- C spectrum contains only one strong emission line, however thallium - antimony spectrum have many spectral lines, giving a possibility to irradiate broader region of the RNA absorption band [4].

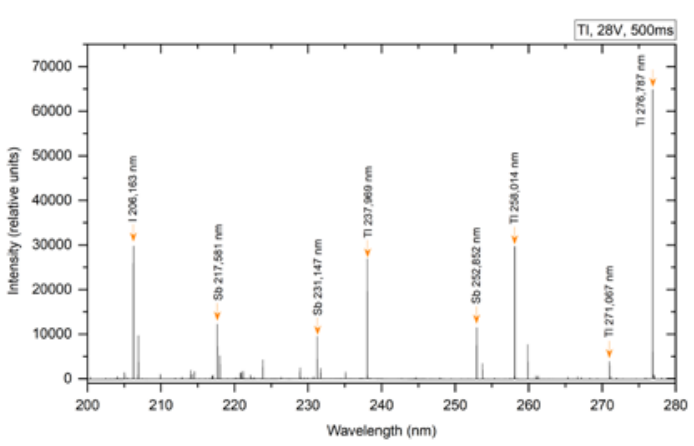

Fig.4. Spectrum example of thallium - antimony light source in the region of $200-280 \mathrm{~nm}$.

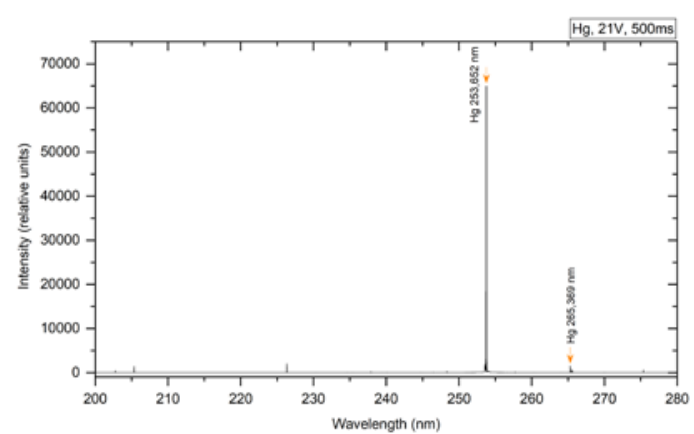

Fig. 5. Spectrum example of mercury light source in the region of 200 $280 \mathrm{~nm}$.

To evaluate and compare the performance of both UV$C$ light sources, bacterial inactivation tests with Gramnegative bacterium - E. coli have been performed to mimic faecal and surface contamination. In general, higher inactivation efficiency was observed with mercury light source, yielding $99 \%$ reduction within 2.26 min of irradiation (Fig. 6, Fig. 7).

At the same time thallium - antimony produced $99 \%$ reduction within 6 minutes and followed a linear reduction pattern all through the irradiation tests (Fig. 7, Fig. 8).

Till some extent this can be explained by different spectral compositions of both light sources. As a result thallium demonstrated comparable efficiency for reduction of cultivable E. coli to mercury UV-C. 


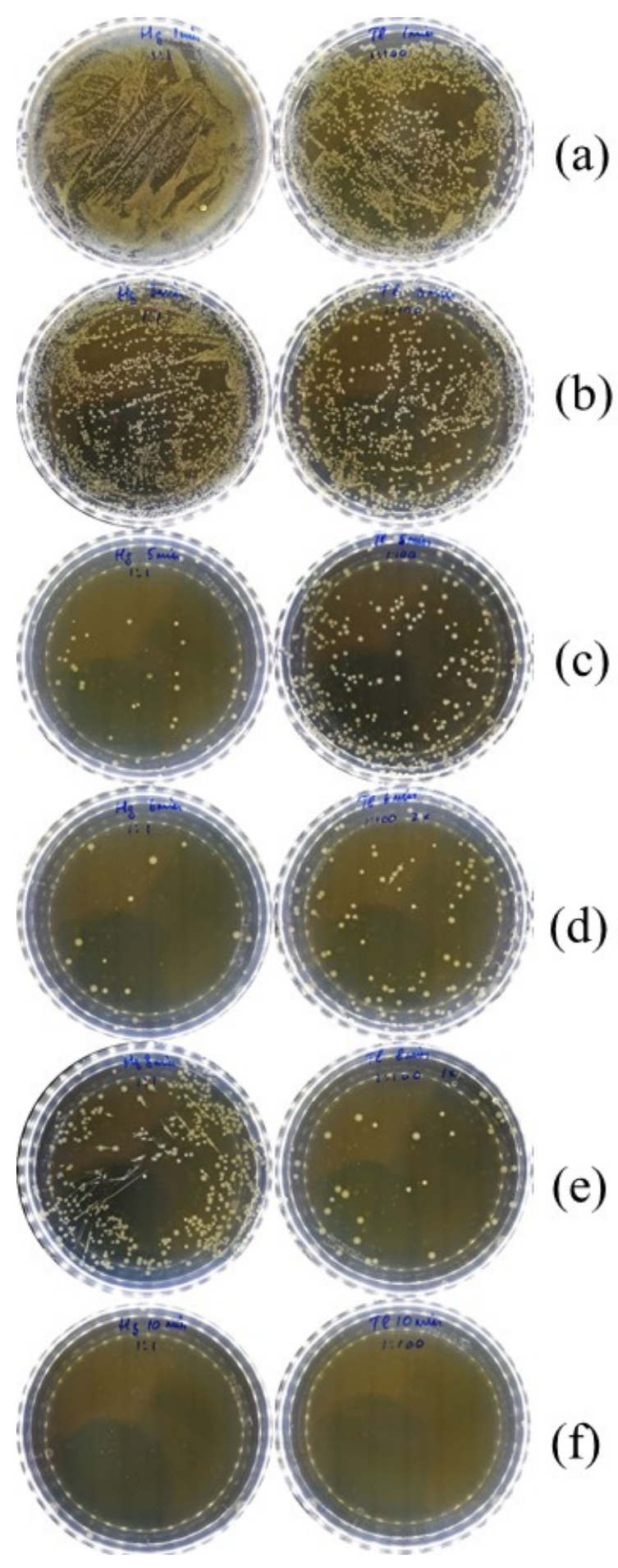

Fig. 6. Decrease in cultivable E. coli (CFU per plate) at various treatment times with mercury (left, no sample dilution) and thallium antimony (right, $10^{2}$ dilution) UV-C light source.(1 min (a), 3 min (b), 5 $\min (\mathrm{c}), 6 \mathrm{~min}(\mathrm{~d}), 8 \mathrm{~min}(\mathrm{e})$ and $10 \mathrm{~min}(\mathrm{f}))$

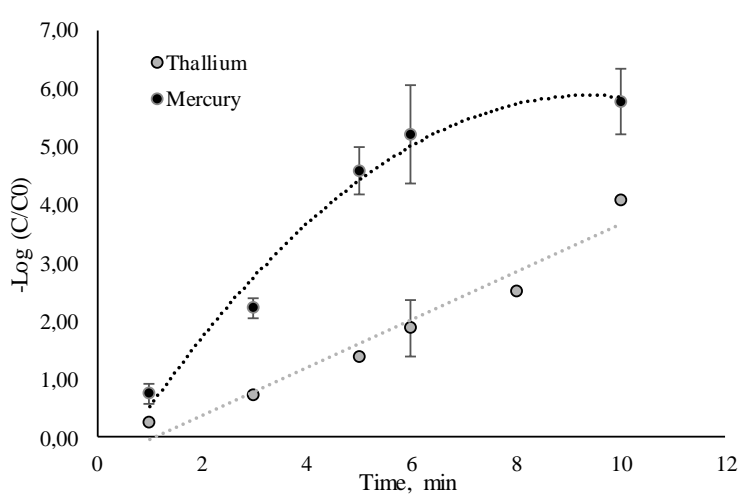

Fig.7. Log-reduction of Escherichia coli CFU as a function of exposure time for thallium - antimony and mercury light sources by equal irradiation conditions.

To date, mercury $254 \mathrm{~nm}$ spectral line irradiation has been demonstrated as efficient source to inactivate both microbial cells and viruses [8, 9]. Within this study even low irradiation doses of thallium - antimony UV-C at spectral region of $200-280 \mathrm{~nm}$ containing many spectral lines showed to be efficient to obtain $99 \%$ reduction of $E$. coli within 6 minutes of contact time. The obtained results demonstrate the potential application of thallium as alternative UV-C light source for microbial and viral contaminant inactivation on surfaces.

\section{CONCLUSIONS}

Mercury UV light source at $254 \mathrm{~nm}$ spectral line demonstrated 99,99\% reduction in less than 5 minutes. The thallium - antimony light source demonstrated lower but still comparable efficiency in tests with Escherichia coli. Thus, UV-C irradiation with non-mercury light sources can be a promising tool for surface and room disinfection.

\section{ACKNOWLEDGEMENTS}

This research was financed from the Latvian State Research Programme VPP-COVID-2020/1-0004 project "Integration of reliable technologies for protection against Covid-19 in healthcare and high risk areas". The authors thank Ms Viktorija Denisova and Mr Ralfs Vevers for technical assistance.

\section{REFERENCES}

[1] N.G. Reed, "The history of ultraviolet germicidal irradiation for air disinfection,” Public health rep., vol 125(1), pp. 15-27, Jan-Feb. 2010.

[2] T. Dai, M.S. Vrahas, C. K. Murray, M.R. Hamblin, "Ultraviolet C irradiation: an alternative antimicrobial approach to localized infections?”, Expert Rev Anti Infect Ther, vol. 10(2), pp. 185-195, Feb. 2012.

[3] M. Heßling, K. Hönes, P. Vatter, C. Lingenfelder, „Ultraviolet irradiation doses for coronavirus inactivation - review and analysis 
of coronavirus photoinactivation studies”, GMS Hyg. Infect. Control, vol.15, 2020, pp. 1-8.

[4] M. Buonanno, G. Randers-Pehrson, A.W. Bigelow, S. Trivedi, F.D. Lowy, H.M. Spotnitz, S.M. Hammer, D.J. Brenner, “207-nm UV light - A promising tool for safe low-cost reduction of surgical site infections. I: In vitro studies”, PLoS One, vol. 8(10), Oct. 2013.

[5] Z. Gavare, A. Svagere, M. Zinge, G. Revalde, V. Fyodorov, Determination of gas temperature of high-frequency lowtemperature electrodeless plasma using molecular spectra of hydrogen and hydroxyl-radical. Journal of Quantitative Spectroscopy \& Radiative Transfer 113 1676-1682 , 2012

[6] G. Revalde et.all, Use of radiaton sources with mercury isotopes for real-time highly sensitive and selectibve benzene determination in air and natural gas by direct differential absorbtion spectrometry with the direct Zeeman effect, Analytica Chimica Acta 887, 172178, 2015.

[7] A.A.Ganeev, Z. Gavare, V.I.Khutorshikov , S.V.Khutorshikov, G. Revalde, A.Skudra G.M.Smirnova, N.R.Stankov, High-frequency electrodeless discharge lamps for atomic absorption, Spectrochemica Acta B, Vol. 58, Issue 5, p. 879-889 (2003).

[8] A. H. Malayeri, M. Mohseni, B. Cairns, J. R. Bolton, „Fluence (UV dose) required to achieve incremental log inactivation of bacteria, protozoa, viruses and algae”, IUVA Newa, 2016.

[9] M. Raeiszadeh, B. Adeli, “A critical review on ultraviolet disinfection systems against COVID-19 outbreak: applicability, validation, and safety considerations”, ASC Photonics, Oct. 2020. 